\title{
Intracoronary Imaging in the Detection of Vulnerable Plaques
}

\author{
Jonathan A. Batty ${ }^{1,2} \cdot$ Shristy Subba $^{1} \cdot$ Peter Luke $^{2} \cdot$ Li Wing Chi Gigi $^{3} \cdot$ \\ Hannah Sinclair $^{1,2} \cdot$ Vijay Kunadian $^{1,2}$
}

Published online: 15 February 2016

(C) The Author(s) 2016. This article is published with open access at Springerlink.com

\begin{abstract}
Coronary artery disease is the result of atherosclerotic changes to the coronary arterial wall, comprising endothelial dysfunction, vascular inflammation and deposition of lipid-rich macrophage foam cells. Certain high-risk atherosclerotic plaques are vulnerable to disruption, leading to rupture, thrombosis and the clinical sequelae of acute coronary syndrome. Though recognised as the gold standard for evaluating the presence, distribution and severity of atherosclerotic lesions, invasive coronary angiography is incapable of identifying non-stenotic, vulnerable plaques that are responsible for adverse cardiovascular events. The recognition of
\end{abstract}

This article is part of the Topical Collection on Interventional Cardiology

Vijay Kunadian

vijay.kunadian@newcastle.ac.uk

Jonathan A. Batty

Jonathan.Batty@newcastle.ac.uk

Shristy Subba

S.Subba1@newcastle.ac.uk

Peter Luke

peter.luke@nuth.nhs.uk

Li Wing Chi Gigi

liwcgigi@gmail.com

Hannah Sinclair

Hannah.Sinclair@newcastle.ac.uk

Institute of Cellular Medicine, Newcastle University, 3rd Floor, William Leech Building, Newcastle Upon Tyne NE2 4HH, UK

2 Freeman Hospital, Newcastle Upon Tyne NHS Foundation Trust, Newcastle Upon Tyne NE7 7DN, UK

3 Chinese University of Hong Kong, Hong Kong SAR, People's Republic of China such limitations has impelled the development of intracoronary imaging technologies, including intravascular ultrasound, optical coherence tomography and near-infrared spectroscopy, which enable the detailed evaluation of the coronary wall and atherosclerotic plaques in clinical practice. This review discusses the present status of invasive imaging technologies; summarises up-to-date, evidence-based clinical guidelines; and addresses questions that remain unanswered with regard to the future of intracoronary plaque imaging.

Keywords Interventional cardiology $\cdot$ Imaging · Coronary artery disease $\cdot$ Intravascular ultrasonography $\cdot$ Near-infrared spectroscopy $\cdot$ Optical coherence tomography

$\begin{array}{ll}\text { Abbreviations } \\ \text { ACS } & \text { Acute coronary syndrome } \\ \text { CAD } & \text { Coronary artery disease } \\ \text { DC } & \text { Dense calcium } \\ \text { FFT } & \text { Fibro-fatty tissue } \\ \text { FT } & \text { Fibrous tissue } \\ \text { IB-IVUS } & \text { Integrated backscatter intravascular ultrasound } \\ \text { IVUS } & \text { Intravascular ultrasound } \\ \text { LCBI } & \text { Lipid-core burden index } \\ \text { LCP } & \text { Lipid-core plaque } \\ \text { MI } & \text { Myocardial infarction } \\ \text { NC } & \text { Necrotic core } \\ \text { NIRS } & \text { Near-infrared spectroscopy } \\ \text { OCT } & \text { Optical coherence tomography } \\ \text { PCI } & \text { Percutaneous coronary intervention } \\ \text { TCFA } & \text { Thin-cap fibroatheroma } \\ \text { VH-IVUS } & \text { Virtual histology intravascular ultrasound }\end{array}$




\section{Introduction}

Despite significant advancements in pharmacological and interventional management, coronary artery disease (CAD) remains the leading cause of morbidity and mortality worldwide $[1,2]$. The first clinical manifestation of CAD is often acute coronary syndrome (ACS), comprising ST-elevation and nonST-elevation acute myocardial infarction (MI) and unstable angina pectoris. CAD comprises a continuum of pathophysiological changes to the intima and media of the coronary arterial wall, characterised by endothelial dysfunction; vascular inflammation; accumulation of lipid, calcium and cellular debris; and in certain plaques, coronary artery stenosis. Longestablished as the gold standard to evaluate the presence, location and extent of stenosis associated with CAD, invasive coronary angiography provides a two-dimensional representation of the coronary lumen but is incapable of visualising the composition of the atherosclerotic plaque [3]. Approximately two thirds of acute coronary events occur due to the rupture of lesions with non-critical $(\leq 50 \%)$ stenosis on angiography and are characterised by specific histological features conferring vulnerability [4-6].

Impelled by the inherent limitations of coronary angiography, complementary imaging modalities have emerged, capable of visualising the components of the arterial wall and characterising coronary plaques. Intravascular ultrasound (IVUS), optical coherence tomography (OCT) and nearinfrared spectroscopy (NIRS) are techniques capable of robust, qualitative and quantitative evaluation of the coronary artery wall, and plaques contained within [7-9]. Emerging clinical evidence suggests that intracoronary imaging is a safe and effective adjunct to angiography, both as a research tool and in clinical practice, providing superior diagnostic precision regarding plaque architecture, composition and severity and enabling incremental risk stratification. However, despite accumulating evidence, guidelines regarding the use of intracoronary imaging for the detection of vulnerable coronary plaques remain limited $[10,11]$.

The purpose of this review is to provide a critical overview of the applications of in vivo intracoronary imaging techniques, in the identification of high-risk, vulnerable atherosclerotic plaques. This review will discuss the available imaging technologies, consider their relative benefits and limitations and explore how the invasive imaging of high-risk, vulnerable plaques may advance in the future.

\section{The Vulnerable Atherosclerotic Plaque}

The concept of the high-risk or vulnerable plaque emerged from landmark, longitudinal studies of patients with STelevation MI. Analysis of serial and pre- and post-infarct angiography demonstrated contrary to popular belief that most infarctions were not caused by severely stenotic lesions [ 5 , 12]. The plaques attributed to causing infarction were moderately sized at baseline. This is explained by expansive remodelling in the arterial wall; the lumen remains uncompromised until the plaque achieves a critical volume. Thus, the degree of stenosis is not the primary determinant of plaque rupture.

A post-mortem, histopathological study examined the hearts of 113 men, 41 of which had thrombosis secondary to vulnerable plaque rupture. Of these, $95 \%$ of lesions had thin fibrous caps, with macrophage infiltrate overlying a lipid-rich pool [6]. The thin-cap fibroatheroma (TCFA) represents a specific morphology of vulnerable plaque, characterised by a thin fibrous layer overlying a large core of lipid-rich necrotic debris and associated with expansive arterial remodelling [13, 14]. TCFA is most frequently observed in the proximal coronary vasculature. Key characteristics conferring vulnerability to rupture include the presence of (i) a thin fibrous cap ( $<65 \mu \mathrm{m})$; (ii) a large, lipid-enriched, necrotic core $(>40 \%$ total plaque volume); (iii) localised macrophage infiltration and inflammation; (iv) vascular remodelling; (v) densely calcified areas (>10\%); and (vi) large plaque volume [15]. Inflammation secondary to shoulder macrophage activation, endothelial denudation, superficial thrombocyte aggregation and haemodynamically significant stenosis $(>90 \%)$ further predispose to rupture and subsequent atherothrombosis [16].

The majority of vulnerable plaques remain clinically silent until the development of acute MI, emphasising the importance of early identification, enabling prognostic stratification and optimisation of management (e.g. risk factor modification; secondary preventative pharmacotherapy).

\section{IVUS}

IVUS Technology Greyscale IVUS uses the amplitude of reflected ultrasound waves to generate an image [17-19]. Many successful applications of IVUS in research have greatly advanced the understanding of the pathophysiology and natural history of coronary atherosclerosis, such as providing the first in vivo evidence of expansive arterial remodelling [20]. IVUS permits detailed evaluation of the coronary artery lumen, wall and plaque area and enables both qualitative and quantitative and pre- and post-intervention assessment of lesion morphology, to a depth of 5-10 $\mathrm{mm}$, with an axial spatial resolution of 100-200 $\mu \mathrm{m}$ at frequencies ranging from 20 to $45 \mathrm{MHz}$. The IVUS catheter is mounted to an automated pullback device, which withdraws the catheter at a pre-set speed (e.g. $0.5 \mathrm{~mm} \mathrm{~s}^{-1}$ ), enabling the acquisition of a cylinder, representative of a length of an artery.

The highly reproducible nature of vessel and plaque measurements using IVUS has been robustly validated [21]. The greyscale image provided by IVUS permits classification of multiple tissues: (i) soft, with echogenicity less than nearby 
adventitia; (ii) calcified, with echogenicity greater than nearby adventitia; (iii) fibrous, with intermediate echogenicity; and (iv) mixed, with several visible acoustic signals $[22,23]$. However, IVUS lacks sensitivity in the identification of lipid-rich plaque (approximately two thirds of all plaques) and has suboptimal spatial resolution to permit a detailed analysis of plaque characteristics, such as visualisation or measurement of fibrous cap thickness (Fig. 1a) [17, 24]. In addition, IVUS cannot reliably identify a thrombus [18]. High-frequency transducers $(>40 \mathrm{MHz}$ ) yield increased resolution, with improved plaque characterisation, at the cost of greater artefact due to reflection of ultrasound from the blood and reduced depth of penetration. Although this may cause confusion
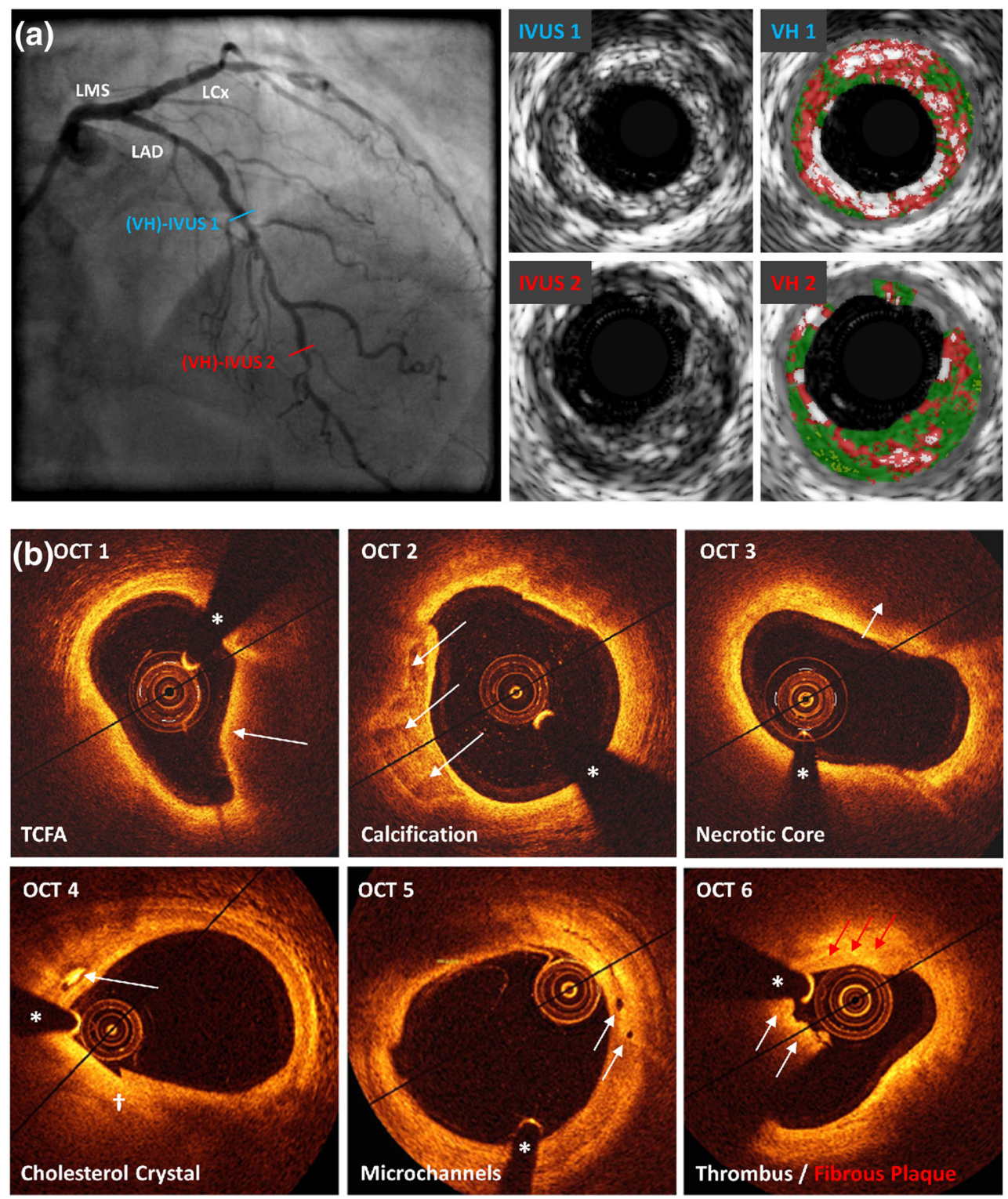

Fig. 1 Examples of intracoronary imaging modalities. a (VH-)IVUS. The coronary angiogram shows the left anterior descending artery (RAO cranial), demonstrating minimal stenosis, but multiple vulnerable plaques are visualised on IVUS. Cross-sectional images demonstrate calcified TCFA (blue line, IVUS1 and VHI) and non-calcified TCFA (red line, IVUS2 and VH2). Keys to VH-IVUS: dark green fibrous tissue, light green fibro-fatty tissue, red necrotic core, white dense calcium. b OCT. Several examples of features associated with plaque vulnerability are presented, including: OCT1 TCFA (arrow indicates thin fibrous cap), OCT2 coronary arterial calcification (arrows indicate well-demarcated calcification), OCT3 necrotic core (arrows indicate lipid pool/necrotic core), OCT4 presence of cholesterol microcrystal (arrow indicates well-demarcated crystal structure), OCT5 microchannels (arrows indicate two separate channels) associated with a nonobstructive lesion and OCT6 (white arrows indicate low-attenuation white thrombus; red arrows indicate highly fibrous plaque). Diag diagonal artery, IVUS intravascular ultrasound, $L A D$ left anterior descending artery, $L C x$ left circumflex artery, $L M S$ left main stem, $O C T$ optical coherence tomography, $O M$ obtuse marginal artery, prob probability, $R A O$ right anterior oblique view, TCFA thin-cap fibroatheroma, $V H$ virtual histology. Asterisk indicates guidewire artefact; dagger indicates seam line artefact 
in interpreting margins between structures (e.g. the lumentissue border), this may be resolved by catheter-guided saline injection [25].

\section{Integrated Backscatter IVUS and Virtual Histology IVUS} Technology Qualitative assessment of plaque morphology using greyscale IVUS requires significant post-processing; the development of plaque characterisation algorithms, such as integrated backscatter IVUS (IB-IVUS) and virtual histology IVUS (VH-IVUS), reduces operator-dependent image interpretation. These techniques augment greyscale IVUS images with a colour map of the plaque architecture, stratified by a tissue type. IB-IVUS uses time domain information extracted directly from the radio-frequency signal to characterise plaque constituents, with high sensitivity and specificity $[26$, 27]. VH-IVUS is performed using either a $20-\mathrm{MHz}$ phased array or a $45-\mathrm{MHz}$ rotational catheter and uses spectral analysis of the frequency and amplitude of ultrasound signals reflected by tissues to characterise plaque components, using an algorithm derived from known tissue types, to detect fibrous tissue (FT, dark green), fibro-fatty tissue (FFT, light green), necrotic core (NC, red) and dense calcium (DC, white; Fig. 1a) [28-31].

The sensitivity and specificity of VH-IVUS is broadly similar to that of IB-IVUS; the degree of concordance between VH-IVUS and in vitro histopathology (following atherectomy) was 87.1-96.5\%, dependent on tissue [32-34]. However, discrepant results exist with regard to the identification of $\mathrm{NC}$; several studies demonstrate poor associations between $\mathrm{VH}-$ IVUS and histological analysis $[35,36]$. This is partly due to the fact that the initial VH-IVUS spectral classification did not differentiate between necrotic and calcified areas, combining the two into calcified-necrotic regions [37].

\section{IVUS, IB-IVUS, VH-IVUS and Vulnerable Plaques}

Definitions The definition of TCFA on IVUS should reflect the histopathological characteristics of TCFA but must respect the limitations of the imaging modality. As such, given the limited resolution of IVUS, the absence of visible fibrous tissue overlying a lipid core implies a cap thickness of $\leq 100-200 \mu \mathrm{m}$. As such, the majority of IVUS studies adopt a simplified definition of TCFA, as a focal necrotic core-rich lesion, without evidence overlying fibrous tissue [38].

IVUS and ACS The multi-centre, PROSPECT (Providing Regional Observations to Study Predictors of Events in the Coronary Tree) cohort study recruited $n=697$ patients with ACS undergoing percutaneous coronary intervention (PCI) to undergo three-vessel greyscale and VH-IVUS [39]. The primary endpoint was a composite of MI, rehospitalisation, cardiac arrest and cardiac-related mortality. A total of 596
TCFA cases were visualised in 313 patients. After a median of 3.4-year follow-up, the rate of the primary endpoint was $20.4 \%$; events during follow-up were attributed to the culprit lesion in only $12.9 \%$. Most of the non-culprit lesions causative of follow-up events were angiographically mild at recruitment (mean \pm standard deviation diameter stenosis 32.3 $\pm 20.6 \%$ ). Multivariate analysis demonstrated that nonculprit lesions associated with events were more likely to be associated with (i) plaque burden of $\geq 70 \%$ (hazard ratio (HR) $5.03,95 \%$ confidence interval (CI) $2.51-10.11 ; p<0.001$ ), (ii) minimal luminal area of $\leq 4.0 \mathrm{~mm}^{2}$ (HR $3.21,95 \% \mathrm{CI}$ $1.61-6.42 ; p=0.001$ ) or (iii) underlying IVUS-TCFA (HR $3.35,95 \%$ CI $1.77-6.36 ; p<0.001)$. However, $48 \%$ of the lesions causing events during follow-up were not imaged at baseline in PROSPECT; IVUS imaging was limited to the proximal and middle portions of the arteries, where the vessel diameter was sufficient to accommodate the IVUS catheter. Crucially, this study suggests that adverse cardiac events have a propensity to occur, with reasonable frequency, at the site of non-culprit lesions.

The VIVA (VH-IVUS and Vulnerable Atherosclerosis) study also prospectively evaluated the association between TCFA and adverse coronary events in $n=170$ patients, with stable angina and ACS, undergoing PCI [28]. All patients underwent a three-vessel VH-IVUS pre- and post-PCI. The primary endpoint consisted of myocardial infarction, unplanned revascularisation or death. At a median of 1.75-year follow-up, the primary endpoint was attributed to 19 lesions (6 culprit and 13 non-culprit) in 16 patients. The same nonculprit factors identified in PROSPECT also associated with the primary endpoint: plaque burden (HR 8.13, $95 \%$ CI 1.63$40.56 ; p=0.011$ ), luminal area (HR 2.91, $95 \%$ CI 1.07-7.91; $p=0.036$ ) and VH-TCFA presence (HR 7.53, $95 \%$ CI 1.12 $50.55 ; p=0.038$ ). This study demonstrates the prognostic significance of IVUS in the identification of patients at high risk of experiencing adverse cardiovascular outcomes.

Recently, the ATHEROREMO-IVUS (European Collaborative Project on Inflammation and Vascular Wall Remodelling in Atherosclerosis-Intravascular Ultrasound) study assessed the value of IVUS in patients with CAD [40 $\bullet$ ]. A total of $n=581$ patients undergoing PCI for stable angina $(n=263)$ and ACS $(n=318)$ underwent VH-IVUS of a non-culprit artery. The primary endpoint comprised a composite of ACS, unplanned revascularisation or death. Of 724 lesions identified by VH-IVUS, 271 (37\%) satisfied criteria for VH-TCFA. At 1-year follow-up, 56 patients had at least one event, 11 had a culprit lesion-related event (which were discounted), 27 had a definite non-culprit lesion-related event and the remaining 18 had an indeterminate event (an event that could not be judged to be either culprit or non-culprit lesion related). The presence of VH-TCFA was independently associated with the composite endpoint (adjusted HR 1.98, $95 \%$ CI $1.09-3.60 ; p=0.026$ ), as was plaque burden 
(adjusted HR 2.90, $95 \%$ CI 1.60-5.25; $p<0.001$ ). In addition, the ATHEROREMO-IVUS was the first study to demonstrate that the presence of VH-TCFA in non-culprit vessels is associated with the hard endpoints of death and ACS at 1 year, not just unplanned rehospitalisation or revascularisation. This study also demonstrated that it may not be necessary to image all three coronary arteries to gain information about the pancoronary vulnerability of the patient.

Together, these landmark studies demonstrate the prognostic potential of VH-IVUS; illustrating how information acquired using this imaging modality regarding plaque burden, minimal luminal area and the presence of TCFA, could be used to incrementally stratify risk in patients with proven CAD.

IVUS and Calcified Plaques Recently, Amano et al. performed a cross-sectional study to evaluate the association between angiographic plaque calcification and vulnerability via the identification of TCFA using VH-IVUS in $n=140$ consecutive patients with ACS [41]. Patients were divided into four groups according to degree of calcification: (i) none (no calcium detected in any lesion; $n=37$ ), spotty (circumferential calcium deposits $<90^{\circ} ; n=65$ ), intermediate (calcium deposits $\geq 90^{\circ}$ but $<180^{\circ} ; n=37$ ) and extensive (calcium deposits $\geq 180^{\circ} ; n=16$ ). The mean number of VH-TCFA in the spotty calcification group was significantly greater than that in the no calcification, intermediate and extensive groups $(0.66 \pm 0.71$ vs. $0.22 \pm 0.42, p<0.01 ; 0.32 \pm 0.48, p<0.05$; and $0.13 \pm 0.34$, $p<0.01$, respectively). In addition, the group with spotty calcification had a significantly greater necrotic core compared to that with angiographic calcification $(p<0.05)$. The presence of angiographic and VH-IVUS calcification correlated strongly $(p<0.05)$. These data suggest that calcified lesions are highly vulnerable; those with spotty or intermediate calcification on VH-IVUS, without angiographic calcification, appear to be more vulnerable than those with angiographic calcification. However, care should be taken with these results, due to the potential issues with the misclassification of necrotic core and dense calcium on the VH-IVUS algorithm.

\section{OCT}

OCT Technology OCT, which uses the reflection of nearinfrared light to generate an image, offers unparalleled spatial resolution $(<10 \mu \mathrm{m}$ axial; $20-40 \mu \mathrm{m}$ lateral $)$ and is a core intravascular imaging modality in clinical practice $[42,43]$. OCT uses a low-coherence, near-infrared light source (centre wavelength, $\lambda_{\mathrm{o}}=1.3 \mu \mathrm{m}$ ) which is directed at and reflected from the vessel wall. The complex principles underlying image generation have been previously described [44]. The OCT catheter contains an optical fibre, which rotates to acquire an image, and is mounted on an automated pullback device, which images the artery in a helical fashion (Fig. 1b). OCT enables high-resolution imaging at the cost of significant artefact due to blood cells, which scatter light and attenuate the image. Therefore, a bloodless field is required for optimal image acquisition.

Two main methods of OCT are in existence: firstgeneration time domain OCT (TD-OCT), which has largely been superseded by second-generation frequency domain OCT (FD-OCT) [43]. TD-OCT generates an image by sequentially measuring isolated near-infrared optical reflections at various depths, with the use of a moving reference mirror. However, this technique is slow (e.g. with pullback speed $1-$ $5 \mathrm{~mm} \mathrm{~s}^{-1}$; image acquisition $\geq 45 \mathrm{~s}$ ) and requires either proximal balloon occlusion (potentially pro-arrhythmogenic) or continuous flushing with iso-osmolar contrast to obtain artefact-free images. Frequency domain OCT uses a nearinfrared source capable of generating $\lambda_{\mathrm{o}}=1.25-1.35 \mu \mathrm{m}$ at a single point, recording reflections at different depths without movement of the reference mirror. Depth profiles, reconstructed using Fourier transformation, permit 10-fold faster acquisition (e.g. with pullback speed $15-40 \mathrm{~mm} \mathrm{~s}^{-1}$; image acquisition 3-5s), without proximal occlusion and minimising contrast injection.

OCT enables high-resolution characterisation of the vascular layers within a healthy artery and can identify morphological changes to the vessel surface associated with high-risk, vulnerable atherosclerotic plaques, including fibrous, lipid-rich and calcified lesions, in addition to red and white thrombus, and macrophages (Fig. 1b) [45, 46]. Potential disadvantages of OCT include a limited depth of penetration $(1-2.5 \mathrm{~mm})$ particularly through lipid-rich lesions, the inability to image the adventitia to assess plaque burden, the requirement for a blood-free field and common imaging artefacts [43, 44].

\section{OCT and Vulnerable Plaques}

Definitions As the axial resolution of OCT is much greater than the diagnostic cut-off for the thin fibrous cap of TCFA, OCT is well placed to identify these in vivo. However, an ongoing debate exists with regard to the precise definition of a TCFA on OCT imaging: there is significant discrepancy between TCFA morphology on histological vs. OCT analysis [47]. In a study of $n=43$ patients with ACS, Tanaka et al. utilised OCT to characterise plaque rupture at the culprit site. Using a cut-off of $70 \mu \mathrm{m}$, the authors demonstrated that $67 \%$ of ruptured plaques demonstrated a thin fibrous cap, in stark comparison to the $\geq 95 \%$ quoted in histopathological studies $[6,48]$. It has been hypothesised that this may be a consequence of the shrinkage of pathological specimens; the true thickness of a TCFA cap may be greater in vivo. Although some studies advocate that the arc of the underlying lipid pool in TCFA should subtend an angle of $\geq 90^{\circ}$, there is no 
consensus that this should be incorporated into the definition of OCT-TCFA.

OCT-TCFA in ACS OCT has been utilised to accurately detect and discriminate between various plaque compositions, predicting peri-PCI complications [46]. A case-control study comparing 26 patients with ST-elevation MI to 16 with stable angina demonstrated that the ACS patients had greater incidence of OCT-TCFA in the culprit lesion ( 85 vs. $13 \%$, $p<0.001)$, with a reduced fibrous cap wall diameter $(57 \pm 12$ vs. $180 \pm 65 \mu \mathrm{m}, p<0.001)$ [49]. This was replicated in similar studies, comparing OCT-TCFA prevalence in ST-elevation vs. non-ST-elevation ACS (78 vs. $49 \%$, respectively; $p=0.008$ ) [50] and in unstable vs. stable angina pectoris (81 vs. $47 \%$, respectively; $p=0.002$ ) [51]. In each study, the average cap thickness was less for the group with greater disease severity, i.e. average thickness in STEMI < NSTEMI < unstable angina $<$ stable angina, respectively [49-51].

In addition, OCT may be used to differentiate between TCFA and eroded or calcified plaques [45]. The features associated with plaque erosion, such as luminal thrombus with absent surface endothelium, have been correlated with sudden cardiac death $[52,53]$. While OCT may correctly identify an irregular luminal surface, the resolution of OCT is inadequate to visualise endothelium $[44,53]$. The use of OCT to detect plaque erosion may be particularly beneficial in ST-elevation MI to guide thrombectomy [54].

OCT and Neoatherosclerosis OCT has permitted new insights into the pathophysiology of neoatherosclerosis, instent restenosis and thrombosis. Initially thought to be associated with the early period after bare-metal stent deployment, evidence of in-stent restenosis has emerged both early and late following insertion of bare-metal and drug-eluting stents and can present as ACS $[55,56]$. OCT imaging following stent deployment demonstrates the transformation of the neointima (which forms as a result of stent neovascularisation) to acquire features of lipid-rich, vulnerable plaque: a process termed neoatherosclerosis [57]. In one study, $n=50$ patients with instent restenosis, and intimal hyperplasia $>50 \%$ of drugeluting stent area, underwent OCT, which demonstrated the presence of TCFA in over half [57]. In a further study, serial OCT observed that approximately one third of patients with homogenous neointimal proliferation demonstrated progression to neoatherosclerosis [58]. As a result, patients with neoatherosclerosis are more likely to develop adverse sequelae, e.g. stent thrombosis and ACS, than those without $[59,60]$.

OCT in Vulnerable Plaque Monitoring The efficacy of pharmacotherapeutic agents in stabilising vulnerable plaques and preventing rupture has been evaluated using OCT. Takarada et al. used serial OCT imaging to compare plaque morphology in $n=40$ patients, allocated to receive either statin therapy or no statin treatment, post-MI [61]. At 9 months, the authors report increases in fibrous cap depth in both groups, with a greater benefit observed in statin-treated patients (192 \pm 41 vs. $25 \pm 8 \mu \mathrm{m}$, respectively; $p<0.001$ ). However, the significance of these changes with regard to clinical outcome is uncertain. A further, prospective trial, evaluating the effect of statin pharmacotherapy on vulnerable plaque morphology in $n=42$ patients with stable angina demonstrated a significant increase in fibrous cap thickness in a statin-treated vs. dietary modification group ( $52 \pm 32$ vs. $2 \pm 22 \mu \mathrm{m}$, respectively; $p<0.001$ ) [62]. No differences were reported in clinical outcome at a median follow-up of 9 months; no patients experienced MI or cardiac-related death, and target vessel revascularisation was similar in both groups (15.4 vs. $18.7 \%$, respectively; $p=0.776$ ). Nishio et al. randomised $n=30$ patients with untreated dyslipidaemia and $\geq 1$ TCFA on baseline OCT to receive statin therapy with or without eicosapentaenoic acid, observing that despite similar levels of low-density lipoprotein at follow-up, patients that received eicosapentaenoic acid experienced greater increases in cap thickness vs. controls (54.8 \pm 27.9 vs. $23.5 \pm 11.6 \mu \mathrm{m}$, respectively; $p<0.0001$ ) [63]. However, while OCT can provide evidence of positive morphological improvements to TCFA, further, longitudinal investigations, with adequate sample sizes, are required to establish if these changes improve clinical outcomes.

\section{NIRS}

NIRS Technology NIRS is a recently developed imaging modality, first tested in vivo in humans in 2001, and is developed for clinical applications over the past decade [64, 65]. NIRS uses a catheter-mounted core of optical fibres to emit and receive diffuse reflectance near-infrared light $\left(\lambda_{\mathrm{o}}=0.8\right.$ $2.5 \mu \mathrm{m})[66,67]$. Similar to OCT, the NIRS catheter contains an optical fibre which rotates ( 240 rotations $\mathrm{min}^{-1}$ ) to acquire an image and is mounted on an automated pullback device. The emitted wavelengths are absorbed in a specific pattern by each component of an atherosclerotic plaque. The diffuse reflectance signals from the tissue are converted to spectra, which undergo algorithmic transformation into a chemogram: a map of the arterial wall, which displays signals suggestive of lipid-core plaque (LCP) [68]. LCP is defined as a plaque $\geq 2 \mathrm{~mm}$ in length, with arterial circumference $\geq 60^{\circ}$ [69]. The $x$-axis represents the position of the catheter relative to the start of the pullback; the $y$-axis indicates the degree of rotation [70, 71]. Red and yellow demonstrate low and high probability of LCP presence, respectively [72, 73]. The chemogram undergoes further processing to yield a summary, block chemogram, providing an interpretation of results for each 2-mm segment of an artery. The same colours are used for the chemogram, which are dependent on the probability of LCP in a given $2-\mathrm{mm}$ region of an artery: red $(p<0.57)$, 
orange $(0.57 \leq p<0.84), \tan (0.84 \leq p<0.98)$ and yellow $(p \geq 0.98)$ [74]. Unlike the aforementioned imaging techniques, NIRS penetrates effectively through calcium and stents. It does not require post-processing nor a bloodless operating field. However, that NIRS provides only compositional insight (i.e. the probability of a lipid-core plaque at a given site) without any quantitative or morphological data (e.g. lumen size, plaque volume) is a limitation [75].

Algorithm Derivation and Validation The algorithm for lipid detection was calibrated using post-mortem coronary arteries [71]. Gardner et al. performed NIRS within 212 segments of a coronary artery (from 84 ex vivo hearts), followed by histopathological analysis of sections taken at 2-mm intervals. The first 33 hearts were used to develop the algorithm; the remaining 51 were used in prospective, double-blind fashion to evaluate NIRS in identifying LCP. The algorithm identified LCP with a receiver-operator characteristic area of $0.80(95 \%$ CI $0.76-0.85$ ) and sensitivity and specificity of 49 and $90 \%$, respectively [71]. The SPECTACL (SPECTroscopic Assessment of Coronary Lipid) study was performed to validate the NIRS algorithm in vivo in $n=106$ patients. This study demonstrated the feasibility and safety of spectral data collection via NIRS, with reasonable sensitivity and specificity [67].

\section{NIRS and Vulnerable Plaques}

LCP, NIRS and ACS The ATHEROREMO-NIRS (European Collaborative Project on Inflammation and Vascular Wall Remodelling in Atherosclerosis-Near-Infrared Spectroscopy) study was performed to assess the prognostic value of NIRS [76••]. NIRS was performed in a non-culprit coronary artery in $n=203$ patients undergoing PCI for stable angina or ACS; LCP within the vessel was measured as the maximal lipid-core burden index (LCBI). Patients were followed up for a median duration of 1 year for a composite endpoint of all-cause mortality, non-fatal ACS, stroke and unplanned coronary revascularisation. Patients with LCBI greater than the median value experienced a significantly greater rate of the composite endpoint vs. patients with LCBI less than the median value ( 16.7 vs. $4.0 \%$, respectively; adjusted HR 4.04, $95 \%$ CI 1.33-12.29; $p=0.01$ ).

The prospective, multi-centre COLOR (Chemometric Observation of Lipid-Core Plaques of Interest in Native Coronary Arteries Registry) registry was established to evaluate the association between the presence of LCP and periprocedural MI [74]. Sixty-two patients with stable preprocedural cardiac biomarkers underwent NIRS prior to PCI. Peri-procedural MI (defined as a cardiac biomarker rise greater than or equal to three times the upper limit of normal) occurred in 7 patients with large LCP (LCBI $\geq 500 ; n=14$ ), compared to 2 patients with lower LCP $(n=48)$ giving a relative risk of 12 (95\% CI 3.3-48; $p=0.0002)$, demonstrating a significant association between high LCP burden and peri-procedural MI.

The recent prospective, multi-centre CANARY (Coronary Assessment by Near-infrared of Atherosclerotic Rupture-prone Yellow) study demonstrated that treatment with PCI reduced LCP burden [77]. Patients experiencing peri-procedural MI had greater LCBI vs. patients without MI (481.5 vs. 371.5 ; $p=0.05$ ). In addition, the effectiveness of a distal protection device in preventing peri-procedural MI was assessed in 31 lesions with $\mathrm{LCBI} \geq 600$. Patients were randomised to receive PCI with distal protection $(n=14)$ vs. PCI without distal protection $(n=17)$. The use of distal protection device had no effect on the rate of peri-procedural MI $(p=0.69)$.

\section{Comparing Established Imaging Techniques}

Several studies have investigated the comparative accuracy of different imaging modalities to detect vulnerable coronary plaque. A recent study by Kini et al. compared IVUS, OCT and NIRS in $n=110$ patients with established CAD, with regard to the prediction of peri-procedural MI [78•]. In patients that developed peri-procedural MI $(n=10)$, OCT-derived minimum fibrous cap thickness was significantly lower compared to those that did not develop MI (55 vs. $90 \mu \mathrm{m}$, respectively; $p<0.01$ ). Both IVUS-measured plaque burden ( $84 \pm 9$ vs. $77 \pm 8 \%$; $p<0.01)$ and NIRS-measured LCBI (556 vs. 339, $p<0.01$ ) were greater in the peri-procedural MI group. The authors performed multivariate logistic regression, identifying OCT cap thickness as the only predictor of peri-procedural MI (odds ratio (OR) $0.91,95 \%$ CI 0.81-0.98; $p=0.04$ ). When removed from the model, the IVUS- and NIRS-derived measures became significant predictors. Thus, OCT-based fibrous cap thickness, suggesting significant plaque vulnerability, is the most significant predictor of peri-procedural MI.

Numerous studies demonstrate that, while the absolute, in vivo diagnostic sensitivity of each imaging technique is favourable, the synergistic use of multiple imaging methodologies may be advantageous. Indeed, to this end, active preclinical and clinical research is ongoing to combine imaging modalities.

Hybridisation of IVUS and OCT Many investigators have speculated that the fusion of IVUS and OCT would prove an optimal imaging strategy. The enhanced resolution of OCT would enable the precise assessment of luminal morphology (i.e. identification of cap thickness, thrombus and plaque erosion), whereas the increased penetration of IVUS would allow the evaluation of plaque burden, architecture and remodelling. The combined usage of IVUS and OCT is proven to improve high-risk plaque detection in multiple studies. Sawada et al. used both VH-IVUS and OCT to detect TCFA in $n=56$ 
patients with established angina. In total, 126 lesions were evaluated, demonstrating that each modality had an adequate absolute sensitivity and specificity for identifying TCFA, which improved when combined [79]. Similarly, Gonzalo et al. demonstrated improved diagnostic precision using $\mathrm{VH}-$ IVUS and OCT [80]. Although single-catheter IVUS-OCT systems are in development, significant barriers remain.

Hybridisation of VH-IVUS and NIRS Although enabling characterisation of plaque architecture, IVUS has limited capability in identifying plaque composition. VH-IVUS analysis provides more data but is subject to underestimation of lipid tissue behind calcified plaques and in stent segments. Brugaletta et al. performed a study comparing VH-IVUS and NIRS in $n=31$ patients with angina, demonstrating poor concordance between the identification of NC on VH-IVUS and LCP on NIRS, despite good sensitivity and specificity for the individual techniques, and the prior association of both entities with adverse events [73]. A study by Pu et al. observed similar findings [81]. Thus, the fusion of these technologies may improve detection of vulnerable plaque. The integration of an ultrasound image to demonstrate morphology, with a chemogram to identify lipid-rich regions, is theoretically advantageous, although evidence is limited regarding the incremental diagnostic utility of this technique. It is currently being used to assess statin-mediated plaque regression in IBIS-3 (Integrated Biomarker and Imaging Study-3) [82].

\section{Other Imaging Techniques}

In addition to established imaging modalities, there are many approaches in active, preclinical and clinical development. We briefly review some of the more prominent technologies.

Coronary Angioscopy Using high-resolution (10-50- $\mu \mathrm{m})$ fibre optics, coronary angioscopy permits the visualisation of the arterial intima and plaque surface [83]. Although capable of assessing superficial characteristics (such as colour), identifying red and white thrombus and detecting localised damage to the vessel wall, (e.g. flaps, fissure, ulceration), angioscopy provides limited insight into plaque morphology. Preliminary data suggests that plaque colour, related to fibrous cap thickness, may be associated with vulnerability (intensely yellow plaques have thinner fibrous caps, predisposing to rupture) [84]. However, angioscopy requires a bloodless field and is highly operator dependent, limiting routine application. As such, coronary angioscopy is primarily limited to research use.

Intravascular MRI The development and application of an intravascular MRI probe, integrated within the tip of a coronary catheter and capable of identifying TCFA in intact, post-mortem coronary arteries, was demonstrated by Schneiderman et al.
[85]. This proof-of-concept device contained both a magnet and coil within a $1.73 \mathrm{~mm}$ diameter. The probe produced axial images, with penetration of $250 \mu \mathrm{m}$, and achieved an estimated resolution of $100 \mu \mathrm{m}$. In preliminary analysis of post-mortem vessels, intravascular MRI identified vulnerable lesions with a sensitivity and specificity of 100 and $89 \%$, respectively. However, long image acquisition times (2.5-4 min), requiring occlusion of the artery, have proven a barrier to translating this technology into in vivo applications.

Raman Spectroscopy Raman spectroscopy identifies organic molecules, using the Raman shift principle, the inelastic scattering of monochromatic light from a laser source, when it reflects off a substance. The unique molecular characteristics of each plaque constituent, particularly lipid and calcium, and their corresponding Raman shift patterns, enable Raman spectroscopy to sensitively detect a coronary plaque [86]. An algorithm, derived from the relative contributions of the independent spectra of the constituents of an atherosclerotic plaque, has been validated [87]. However, intracoronary applications have numerous limitations, such as the small proportion of photons that become shifted, resulting in a poor signal-to-noise ratio and limited tissue penetration.

Near-Infrared Fluorescence Molecular Imaging Using technology similar to NIRS, intravascular near-infrared fluorescence molecular imaging utilises the phenomenon of molecular fluorescence to identify specific regions of arterial inflammation and injury [88]. Near-infrared fluorescence (NIRF) permits a targeted approach to the identification of plaque components (e.g. lipid core). Preclinical, proof-ofconcept studies have used NIRF catheters, in conjunction with a cysteine protease imaging reporter, for in vivo intracoronary imaging in animal models. Jaffer et al. report high-resolution spatial mapping of arterial inflammation, superimposed on IVUS imaging, to identify high-risk plaque regions [89].

A comparison of all imaging technologies described in this review is presented in Table 1.

\section{Clinical Guidelines}

Evidence-based clinical practice guidelines, regarding the usage of intracoronary imaging, have been developed by the ACC/ AHA/SCAI and ESC/EACTS. The 2011 North American guidelines recommend the use of IVUS which is reasonable for the assessment of (i) angiographically indeterminate left main CAD (class IIa, level of evidence B) and (ii) non-left main coronary arteries with angiographically intermediate stenoses (50-70 \%; class IIb, level of evidence B) [90]. The guidelines suggest other situations in which the IVUS may have clinical utility, such as in the evaluation of the aetiology of stent restenosis (class IIa, level of evidence $\mathrm{C}$ ) and thrombosis (class IIb, 
Table 1 Comparison of imaging technologies for the detection of vulnerable coronary plaques

\begin{tabular}{|c|c|c|c|c|c|c|c|c|c|}
\hline \multirow{2}{*}{$\begin{array}{l}\text { Imaging } \\
\text { technique }\end{array}$} & \multirow{2}{*}{$\begin{array}{l}\text { Imaging } \\
\text { technology }\end{array}$} & \multirow{2}{*}{$\begin{array}{l}\text { Wavelength } \\
(\mu \mathrm{m})\end{array}$} & \multirow{2}{*}{$\begin{array}{l}\text { Penetration } \\
(\mathrm{mm})\end{array}$} & \multirow{2}{*}{$\begin{array}{l}\text { Resolution } \\
(\mu \mathrm{m})\end{array}$} & \multicolumn{5}{|c|}{ Clinical utility in detection of vulnerable plaque feature } \\
\hline & & & & & $\begin{array}{l}\text { Fibrous } \\
\text { cap }\end{array}$ & $\begin{array}{l}\text { Lipid } \\
\text { core }\end{array}$ & Inflammation & Calcium & Thrombus \\
\hline $\begin{array}{l}\text { Coronary } \\
\quad \text { Angiography }\end{array}$ & X-ray & $0.00001-0.01$ & 0.0 & $>500$ & - & - & - & + & +++ \\
\hline$(\mathrm{IB} / \mathrm{VH}-)_{\mathrm{IVUS}}^{\mathrm{a}}$ & Ultrasound & $35-80$ & 10.0 & $100-200$ & + & ++ & - & +++ & - \\
\hline $\mathrm{OCT}^{\mathrm{a}}$ & Infrared & 1.3 & $1.0-2.5$ & $<10$ & +++ & +++ & + & ++ & ++ \\
\hline NIRS & Near-infrared & $0.8-2.5$ & $1.0-2.0$ & N/A & - & +++ & - & - & - \\
\hline Raman spectroscopy & Near-infrared & $0.75-1.0$ & $1.0-2.0$ & N/A & - & +++ & - & - & - \\
\hline IV-MRI & MRI & N/A & 0.25 & 100 & + & ++ & ++ & ++ & + \\
\hline Angioscopy & Optical & $0.4-0.7$ & 0.0 & $10-50$ & + & + & - & - & +++ \\
\hline Thermography & Infrared & $0.8-2.5$ & 1.0 & 500 & - & - & +++ & - & - \\
\hline
\end{tabular}

$-=$ not possible $+=$ adequate $++=$ good $+++=$ excellent

$I B$ integrated backscatter, $I V-M R I$ intravascular MRI, IVUS intravascular ultrasound, $m m$ millimetres, $N / A$ not applicable, NIRS near-infrared spectroscopy, OCT optical coherence tomography, $\mu m$ micrometres

${ }^{\text {a }}$ In routine clinical use

level of evidence C). The routine use of IVUS for lesion evaluation, when PCI is otherwise not intended, is not recommended (class III). Although the guideline acknowledges OCT, it does not provide recommendations for usage outside of clinical research. No recommendations are made regarding other imaging modalities. The 2014 European guidelines advocate IVUS in selected patients (i) to optimise stent placement (class IIa, level of evidence B) and (ii) to assess severity and optimise treatment of unprotected left main lesions (class IIa, level of evidence B) [91]. The guideline also advises consideration of IVUS and/or OCT to detect stent-related mechanical problems (e.g. restenosis, thrombosis; class IIa, level of evidence C) and OCT in selected patients to optimise stent implantation (class IIb, level of evidence C). No recommendations are made regarding other imaging modalities. Both recommendations advocate the need for further clinical research to demonstrate that the use of intracoronary imaging techniques improves clinical outcomes.

\section{Conclusion}

The development of invasive intracoronary imaging, exemplified by IVUS, OCT and NIRS, has revolutionised an understanding of the pathophysiology of coronary artery disease. Translation into the cardiac catheterisation lab has permitted detailed, in vivo identification and evaluation of high-risk, vulnerable coronary plaques in both clinical research and cardiology practice. Of the available array of imaging modalities, each has distinct advantages and disadvantages: VH-IVUS enables precise characterisation of plaque architecture but lacks spatial resolution. OCT permits high-resolution measurement of the fibrous cap but has limited penetration through the arterial wall. NIRS robustly detects lipid cores but provides limited quantitative data regarding the coronary lumen or plaque morphology. Hybrid approaches negate these limitations and are gaining traction in early clinical studies. Numerous novel imaging technologies are under development. However, while improved image quality is imperative, nascent techniques must add value to existing approaches. Robust clinical trials are required to evaluate whether invasive intracoronary imaging improves patient outcomes.

\section{Compliance with Ethical standards}

Conflict of Interest Jonathan A. Batty, Shristy Subba, Peter Luke, Li Wing Chi Gigi, Hannah Sinclair and Vijay Kunadian declare that they have no conflict of interest.

Human and Animal Rights and Informed Consent This article does not contain any studies with human or animal subjects performed by any of the authors.

Open Access This article is distributed under the terms of the Creative Commons Attribution 4.0 International License (http:// creativecommons.org/licenses/by/4.0/), which permits unrestricted use, distribution, and reproduction in any medium, provided you give appropriate credit to the original author(s) and the source, provide a link to the Creative Commons license, and indicate if changes were made.

\section{References}

Papers of particular interest, published recently, have been highlighted as:

- Of importance

-• Of major importance

1. Mozaffarian D, Benjamin EJ, Go AS, et al. Heart disease and stroke statistics-2015 update: a report from the American Heart Association. Circulation. 2015;131(4):e29-322. 
2. Nichols M, Townsend N, Scarborough P, et al. Cardiovascular disease in Europe 2014: epidemiological update. Eur Heart J. 2014;35(42):2950-9.

3. Ryan TJ. The coronary angiogram and its seminal contributions to cardiovascular medicine over five decades. Circulation. 2002;106(6):752-6.

4. Topol EJ, Nissen SE. Our preoccupation with coronary luminology. The dissociation between clinical and angiographic findings in ischemic heart disease. Circulation. 1995;92(8):2333-42.

5. Little WC, Constantinescu M, Applegate RJ, et al. Can coronary angiography predict the site of a subsequent myocardial infarction in patients with mild-to-moderate coronary artery disease? Circulation. 1988;78(5 Pt 1):1157-66.

6. Burke AP, Farb A, Malcom GT, et al. Coronary risk factors and plaque morphology in men with coronary disease who died suddenly. N Engl J Med. 1997;336(18):1276-82.

7. Mintz GS. Clinical utility of intravascular imaging and physiology in coronary artery disease. J Am Coll Cardiol. 2014;64(2):207-22.

8. Hayat U, Thondapu V, Asrar Ul Haq M, et al. Optical coherence tomography to evaluate coronary stent implantation and complications. Coron Artery Dis. 2015;26 Suppl 1:e55-68.

9. Konishi A, Shinke T, Otake H, et al. Serial optical coherence tomography evaluation at 6,12 , and 24 months after Biolimus A9eluting biodegradable polymer-coated stent implantation. Can J Cardiol. 2015;31(8):980-8.

10. Groves EM, Seto AH, Kern MJ. Invasive testing for coronary artery disease: FFR, IVUS, OCT, NIRS. Cardiol Clin. 2014;32(3):40517.

11. Jegere S, Narbute I, Erglis A. Use of intravascular imaging in managing coronary artery disease. World J Cardiol. 2014;6(6):393-404.

12. Ambrose JA, Tannenbaum MA, Alexopoulos D, et al. Angiographic progression of coronary artery disease and the development of myocardial infarction. J Am Coll Cardiol. 1988;12(1):56-62.

13. Miyamoto $\mathrm{Y}$, Okura H, Kume T, et al. Plaque characteristics of thincap fibroatheroma evaluated by OCT and IVUS. JACC Cardiovasc Imaging. 2011;4(6):638-46.

14. Varnava AM, Mills PG, Davies MJ. Relationship between coronary artery remodeling and plaque vulnerability. Circulation. 2002;105(8):939-43.

15. Finn AV, Nakano M, Narula J, et al. Concept of vulnerable/unstable plaque. Arterioscler Thromb Vasc Biol. 2010;30(7):1282-92.

16. Bentzon JF, Otsuka F, Virmani R, et al. Mechanisms of plaque formation and rupture. Circ Res. 2014;114(12):1852-66.

17. Fujii $\mathrm{K}$, Hao H, Ohyanagi $\mathrm{M}$, et al. Intracoronary imaging for detecting vulnerable plaque. Circ J. 2013;77(3):588-95.

18. Maehara A, Mintz GS, Weissman NJ. Advances in intravascular imaging. Circ Cardiovasc Interv. 2009;2(5):482-90.

19. Yock PG, Linker DT, Angelsen BA. Two-dimensional intravascular ultrasound: technical development and initial clinical experience. J Am Soc Echocardiogr. 1989;2(4):296-304.

20. Glagov S, Weisenberg E, Zarins CK, et al. Compensatory enlargement of human atherosclerotic coronary arteries. N Engl J Med. 1987;316(22):1371-5.

21. Nissen SE, Gurley JC, Grines CL, et al. Intravascular ultrasound assessment of lumen size and wall morphology in normal subjects and patients with coronary artery disease. Circulation. 1991;84(3): 1087-99.

22. Garcìa-Garcìa HM, Gogas BD, Serruys PW, et al. IVUS-based imaging modalities for tissue characterization: similarities and differences. Int J Cardiovasc Imaging. 2011;27(2):215-24.

23. Mintz GS, Nissen SE, Anderson WD, et al. American College of Cardiology Clinical Expert Consensus Document on Standards for Acquisition, Measurement and Reporting of Intravascular Ultrasound Studies (IVUS). A report of the American College of
Cardiology Task Force on Clinical Expert Consensus Documents. J Am Coll Cardiol. 2001;37(5):1478-92.

24. Prati F, Arbustini E, Labellarte A, et al. Intravascular ultrasound insights into plaque composition. Z Kardiol. 2000;89 Suppl 2: 117-23.

25. Honye J, Saito S, Takayama T, et al. Clinical utility of negative contrast intravascular ultrasound to evaluate plaque morphology before and after coronary interventions. Am J Cardiol. 1999;83(5):687-90.

26. Kawasaki M, Takatsu $H$, Noda $T$, et al. In vivo quantitative tissue characterization of human coronary arterial plaques by use of integrated backscatter intravascular ultrasound and comparison with angioscopic findings. Circulation. 2002;105(21):2487-92.

27. Okubo M, Kawasaki M, Ishihara Y, et al. Development of integrated backscatter intravascular ultrasound for tissue characterization of coronary plaques. Ultrasound Med Biol. 2008;34(4):655-63.

28. Calvert PA, Obaid DR, O'Sullivan M, et al. Association between IVUS findings and adverse outcomes in patients with coronary artery disease: the VIVA (VH-IVUS in Vulnerable Atherosclerosis) Study. JACC Cardiovasc Imaging. 2011;4(8): 894-901.

29. Suh WM, Seto AH, Margey RJ, et al. Intravascular detection of the vulnerable plaque. Circ Cardiovasc Imaging. 2011;4(2):169-78.

30. Pundziute G, Schuijf JD, Jukema JW, et al. Head-to-head comparison of coronary plaque evaluation between multislice computed tomography and intravascular ultrasound radiofrequency data analysis. JACC Cardiovasc Interv. 2008;1(2):176-82.

31. García-García HM, Mintz GS, Lerman A, et al. Tissue characterisation using intravascular radiofrequency data analysis: recommendations for acquisition, analysis, interpretation and reporting. EuroIntervention. 2009;5(2):177-89.

32. Nasu K, Tsuchikane E, Katoh O, et al. Accuracy of in vivo coronary plaque morphology assessment: a validation study of in vivo virtual histology compared with in vitro histopathology. J Am Coll Cardiol. 2006;47(12):2405-12.

33. Watson RJ, McLean CC, Moore MP, et al. Classification of arterial plaque by spectral analysis of in vitro radio frequency intravascular ultrasound data. Ultrasound Med Biol. 2000;26(1):73-80.

34. Nair A, Kuban BD, Tuzcu EM, et al. Coronary plaque classification with intravascular ultrasound radiofrequency data analysis. Circulation. 2002;106(17):2200-6.

35. Brugaletta S, Cola C, Martin-Yuste V, et al. Qualitative and quantitative accuracy of ultrasound-based virtual histology for detection of necrotic core in human coronary arteries. Int J Cardiovasc Imaging. 2014;30(3):469-76.

36. Granada JF, Wallace-Bradley D, Win HK, et al. In vivo plaque characterization using intravascular ultrasound-virtual histology in a porcine model of complex coronary lesions. Arterioscler Thromb Vasc Biol. 2007;27(2):387-93.

37. Nair A, Kuban BD, Obuchowski N, et al. Assessing spectral algorithms to predict atherosclerotic plaque composition with normalized and raw intravascular ultrasound data. Ultrasound Med Biol. 2001;27(10):1319-31.

38. Rodriguez-Granillo GA, Garcia-Garcia HM, Mc Fadden EP, et al. In vivo intravascular ultrasound-derived thin-cap fibroatheroma detection using ultrasound radiofrequency data analysis. J Am Coll Cardiol. 2005;46(11):2038-42.

39. Stone GW, Maehara A, Lansky AJ, et al. A prospective naturalhistory study of coronary atherosclerosis. N Engl J Med. 2011;364(3):226-35.

40.• Cheng JM, Garcia-Garcia HM, de Boer SP, et al. In vivo detection of high-risk coronary plaques by radiofrequency intravascular ultrasound and cardiovascular outcome: results of the ATHEROREMOIVUS study. Eur Heart J. 2014;35(10):639-47. ATHEROREMOIVUS was the first study to demonstrate that VH-TCFA in non- 
culprit vessels is associated with the hard cardiovascular endpoints at 1 year.

41. Amano H, Ikeda T, Toda M, et al. Assessment of angiographic coronary calcification and plaque composition in virtual histology intravascular ultrasound. J Interv Cardiol. 2015;28(2):205-14.

42. Jang IK, Bouma BE, Kang DH, et al. Visualization of coronary atherosclerotic plaques in patients using optical coherence tomography: comparison with intravascular ultrasound. J Am Coll Cardiol. 2002;39(4):604-9.

43. Roleder T, Jąkała J, Kałuża GL, et al. The basics of intravascular optical coherence tomography. Postepy Kardiol Interwencyjnej. 2015;11(2):74-83

44. Sinclair $\mathrm{H}$, Bourantas $\mathrm{C}$, Bagnall A, et al. OCT for the identification of vulnerable plaque in acute coronary syndrome. J Am Coll Cardiol Img. 2015;8(2):198-209.

45. Kubo T, Tanaka A, Ino Y, et al. Assessment of coronary atherosclerosis using optical coherence tomography. J Atheroscler Thromb. 2014;21(9):895-903.

46. Kubo T, Tanaka A, Kitabata H, et al. Application of optical coherence tomography in percutaneous coronary intervention. Circ J. 2012;76(9):2076-83.

47. Tearney GJ, Regar E, Akasaka T, et al. Consensus standards for acquisition, measurement, and reporting of intravascular optical coherence tomography studies: a report from the International Working Group for Intravascular Optical Coherence Tomography Standardization and Validation. J Am Coll Cardiol. 2012;59(12): 1058-72.

48. Tanaka A, Imanishi T, Kitabata H, et al. Morphology of exertiontriggered plaque rupture in patients with acute coronary syndrome: an optical coherence tomography study. Circulation. 2008;118(23): 2368-73.

49. Kubo T, Imanishi T, Kashiwagi M, et al. Multiple coronary lesion instability in patients with acute myocardial infarction as determined by optical coherence tomography. Am J Cardiol. 2010;105(3):318-22.

50. Ino Y, Kubo T, Tanaka A, et al. Difference of culprit lesion morphologies between st-segment elevation myocardial infarction and nonST-segment elevation acute coronary syndrome: an optical coherence tomography study. JACC Cardiovasc Interv. 2011;4(1):76-82.

51. Tian J, Hou J, Xing L, et al. Significance of intraplaque neovascularisation for vulnerability: optical coherence tomography study. Heart. 2012;98(20):1504-9.

52. Tavora F, Cresswell N, Li L, et al. Sudden coronary death caused by pathologic intimal thickening without atheromatous plaque formation. Cardiovasc Pathol. 2011;20(1):51-7.

53. Bentzon JF, Otsuka F, Virmani R, et al. Mechanisms of plaque formation and rupture. Circ Res. 2014;114(12):1852-66.

54. Prati F, Uemura S, Souteyrand G, et al. OCT-based diagnosis and management of STEMI associated with intact fibrous cap. JACC Cardiovasc Imaging. 2013;6(3):283-7.

55. Kimura T, Yokoi H, Nakagawa Y, et al. Three-year follow-up after implantation of metallic coronary-artery stents. N Engl J Med. 1996;334(9):561-6.

56. Nakagawa $Y$, Kimura $T$, Morimoto $T$, et al. Incidence and risk factors of late target lesion revascularization after sirolimuseluting stent implantation (3-year follow-up of the j-Cypher Registry). Am J Cardiol. 2010;106(3):329-36.

57. Takano M, Yamamoto M, Inami S, et al. Appearance of lipid-laden intima and neovascularization after implantation of bare-metal stents extended late-phase observation by intracoronary optical coherence tomography. J Am Coll Cardiol. 2009;55(1):26-32.

58. Kim JS, Hong MK, Shin DH, et al. Quantitative and qualitative changes in DES-related neointimal tissue based on serial OCT. JACC Cardiovasc Imaging. 2012;5(11):1147-55.
59. Kang SJ, Mintz GS, Akasaka T, et al. Optical coherence tomographic analysis of in-stent neoatherosclerosis after drug-eluting stent implantation. Circulation. 2011;123(25):2954-63.

60. Lee SY, Shin DH, Mintz GS, et al. Optical coherence tomographybased evaluation of in-stent neoatherosclerosis in lesions with more than $50 \%$ neointimal cross-sectional area stenosis. EuroIntervention. 2013;9(8):945-51.

61. Takarada S, Imanishi T, Kubo T, et al. Effect of statin therapy on coronary fibrous-cap thickness in patients with acute coronary syndrome: assessment by optical coherence tomography study. Atherosclerosis. 2009;202(2):491-7.

62. Hattori K, Ozaki Y, Ismail TF, et al. Impact of statin therapy on plaque characteristics as assessed by serial OCT, grayscale and integrated backscatter-IVUS. JACC Cardiovasc Imaging. 2012;5(2):169-77.

63. Nishio R, Shinke T, Otake H, et al. Stabilizing effect of combined eicosapentaenoic acid and statin therapy on coronary thin-cap fibroatheroma. Atherosclerosis. 2014;234(1):114-9.

64. Moreno PR, Muller JE. Identification of high-risk atherosclerotic plaques: a survey of spectroscopic methods. Curr Opin Cardiol. 2002;17(6):638-47.

65. Caplan JD, Waxman S, Nesto RW, et al. Near-infrared spectroscopy for the detection of vulnerable coronary artery plaques. J Am Coll Cardiol. 2006;47(8 Suppl):C92-6.

66. Jaguszewski M, Klingenberg R, Landmesser U. Intracoronary nearinfrared spectroscopy (NIRS) imaging for detection of lipid content of coronary plaques: current experience and future perspectives. Curr Cardiovasc Imaging Rep. 2013;6:426-30.

67. Waxman S, Dixon SR, L'Allier P, et al. In vivo validation of a catheter-based near-infrared spectroscopy system for detection of lipid core coronary plaques: initial results of the SPECTACL study. JACC Cardiovasc Imaging. 2009;2(7):858-68.

68. de Boer SP, Brugaletta S, Garcia-Garcia HM, et al. Determinants of high cardiovascular risk in relation to plaque-composition of a nonculprit coronary segment visualized by near-infrared spectroscopy in patients undergoing percutaneous coronary intervention. Eur Heart J. 2014;35(5):282-9.

69. Brugaletta S, Sabaté M. Assessment of plaque composition by intravascular ultrasound and near-infrared spectroscopy: from PROSPECT I to PROSPECT II. Circ J. 2014;78(7):1531-9.

70. Kang SJ, Mintz GS, Pu J, et al. Combined IVUS and NIRS detection of fibroatheromas: histopathological validation in human coronary arteries. JACC Cardiovasc Imaging. 2015;8(2):184-94.

71. Gardner CM, Tan H, Hull EL, et al. Detection of lipid core coronary plaques in autopsy specimens with a novel catheter-based near-infrared spectroscopy system. J Am Coll Cardiol Img. 2008;1(5): 638-48.

72. Fabris E, Kilic ID, Caiazzo G, et al. Three-vessel coronary artery disease evaluation by multimodality imaging with near-infrared spectroscopy (NIRS) plus intravascular ultrasound (IVUS) and optical coherence tomography (OCT). Int J Cardiol. 2015;180:21-9.

73. Brugaletta S, Garcia-Garcia HM, Serruys PW, et al. NIRS and IVUS for characterization of atherosclerosis in patients undergoing coronary angiography. JACC Cardiovasc Imaging. 2011;4(6):647-55.

74. Goldstein JA, Maini B, Dixon SR, et al. Detection of lipid-core plaques by intracoronary near-infrared spectroscopy identifies high risk of periprocedural myocardial infarction. Circ Cardiovasc Interv. 2011;4(5):429-37.

75. Negi SI, Didier R, Ota H, et al. Role of near-infrared spectroscopy in intravascular coronary imaging. Cardiovase Revasc Med. 2015;16(5):299-305.

76.• Oemrawsingh RM, Cheng JM, García-García HM, et al. Nearinfrared spectroscopy predicts cardiovascular outcome in patients with coronary artery disease. J Am Coll Cardiol. 2014;64(23): 2510-8. ATHEROREMO-NIRS demonstrated the association 
of high LCBI with incident adverse cardiovascular events, in a large number of patients.

77. Stone GW, Maehara A, Muller JE, et al. Plaque characterization to inform the prediction and prevention of periprocedural myocardial infarction during percutaneous coronary intervention: the CANARY Trial (Coronary Assessment by Near-infrared of Atherosclerotic Rupture-prone Yellow). JACC Cardiovasc Interv. 2015;8(7):927-36.

78. Kini AS, Motoyama S, Vengrenyuk Y, et al. Multimodality intravascular imaging to predict periprocedural myocardial infarction during percutaneous coronary intervention. JACC Cardiovasc Interv. 2015;8(7):937-45. This is one of the only studies to use all three core imaging modalities (IVUS, OCT and NIRS) in patients with coronary artery disease for the prediction of peri-procedural MI.

79. Sawada T, Shite J, Garcia-Garcia HM, et al. Feasibility of combined use of intravascular ultrasound radiofrequency data analysis and optical coherence tomography for detecting thin-cap fibroatheroma. Eur Heart J. 2008;29(9):1136-46.

80. Gonzalo N, Garcia-Garcia HM, Regar E, et al. In vivo assessment of high-risk coronary plaques at bifurcations with combined intravascular ultrasound and optical coherence tomography. JACC Cardiovasc Imaging. 2009;2(4):473-82.

81. Pu J, Mintz GS, Brilakis ES, et al. In vivo characterization of coronary plaques: novel findings from comparing greyscale and virtual histology intravascular ultrasound and near-infrared spectroscopy. Eur Heart J. 2012;33(3):372-83.

82. Simsek C, Garcia-Garcia HM, van Geuns RJ, et al. The ability of high dose rosuvastatin to improve plaque composition in nonintervened coronary arteries: rationale and design of the Integrated Biomarker and Imaging Study-3 (IBIS-3). EuroIntervention. 2012;8(2):235-41.
83. Ishibashi F, Aziz K, Abela GS, et al. Update on coronary angioscopy: review of a 20 -year experience and potential application for detection of vulnerable plaque. J Interv Cardiol. 2006;19(1):17-25.

84. Kubo T, Imanishi T, Takarada S, et al. Implication of plaque color classification for assessing plaque vulnerability: a coronary angioscopy and optical coherence tomography investigation. JACC Cardiovasc Interv. 2008;1(1):74-80.

85. Schneiderman J, Wilensky RL, Weiss A, et al. Diagnosis of thin-cap fibroatheromas by a self-contained intravascular magnetic resonance imaging probe in ex vivo human aortas and in situ coronary arteries. J Am Coll Cardiol. 2005;45(12):1961-9.

86. Brennan 3rd JF, Romer TJ, Lees RS, et al. Determination of human coronary artery composition by Raman spectroscopy. Circulation. 1997;96(1):99-105.

87. Buschman HP, Motz JT, Deinum G, et al. Diagnosis of human coronary atherosclerosis by morphology-based Raman spectroscopy. Cardiovasc Pathol. 2001;10(2):59-68.

88. Thukkani AK, Jaffer FA. Intravascular near-infrared fluorescence molecular imaging of atherosclerosis. Am J Nucl Med Mol Imaging. 2013;3(3):217-31.

89. Jaffer FA, Calfon MA, Rosenthal A, et al. Two-dimensional intravascular near-infrared fluorescence molecular imaging of inflammation in atherosclerosis and stent-induced vascular injury. J Am Coll Cardiol. 2011;57(25):2516-26.

90. Levine GN, Bates ER, Blankenship JC, et al. ACCF/AHA/SCAI Guideline for Percutaneous Coronary Intervention: a report of the American College of Cardiology Foundation/American Heart Association Task Force on Practice Guidelines and the Society for Cardiovascular Angiography and Interventions. Circulation. 2011;124(23):e574-651.

91. Windecker S, Kolh P, Alfonso F, et al. ESC/EACTS guidelines on myocardial revascularization. Eur Heart J. 2014;35(37):2541-619. 